\title{
Investigation of the antigenicity and protective efficacy of Leishmania promastigote membrane antigens in search of potential diagnostic and vaccine candidates against visceral leishmaniasis
}

\author{
Sarfaraz Ahmad Ejazi ${ }^{1+}$, Smriti Ghosh ${ }^{1,2+}$, Anirban Bhattacharyya ${ }^{1}$, Mohd Kamran$^{1}$, Sonali Das ${ }^{1}$,
} Sudipta Bhowmick ${ }^{1,3}$, Mehebubar Rahaman ${ }^{4}$, Rama Prosad Goswami ${ }^{4}$ and Nahid $\mathrm{Ali}^{1 *}$

\begin{abstract}
Background: Visceral leishmaniasis $(\mathrm{VL})$, is a parasitic disease that causes serious medical consequences if treatment is delayed. Despite a decline in the number of VL cases in the Indian subcontinent, the commencement of the disease in newer areas continues to be a major concern. Although serological diagnosis mainly by immunochromatographic tests has been found to be effective, a test of cure in different phases of treatment is still desired. Even though a good prophylactic response has been obtained in murine models by a number of vaccine candidates, few have been proposed for human use.

Methods: In this study, nine antigenic components $(31,34,36,45,51,63,72,91$ and 97 kDa) of Leishmania promastigote membrane antigens ( $\mathrm{LAg}$ ), were electroeluted and evaluated through ELISA to diagnose and distinguish active $V L$ from one month cured and six months post-treatment patients. Further, to investigate the immunogenicity of electroeluted proteins, human PBMCs of cured VL patients were stimulated with 31, 34, 51, 63, 72 and 91 kDa proteins.

Results: We found that 34 and $51 \mathrm{kDa}$ proteins show $100 \%$ sensitivity and specificity with healthy controls and other diseases. After six months post-treatment, antibodies to 72 and $91 \mathrm{kDa}$ antigens show a significant decline to almost normal levels. This suggests that 34 and $51 \mathrm{kDa}$ proteins are efficient in diagnosis, whereas 72 and $91 \mathrm{kDa}$ proteins may be used to monitor treatment outcome. In another assay, 51 and $63 \mathrm{kDa}$ proteins demonstrated maximum ability to upregulate IFN- $\gamma$ and IL-12 with minimum induction of IL-10 and TGF- $\beta$. The results indicating that 51 and $63 \mathrm{kDa}$ proteins could be strong candidates for human immunization against VL. In contrast, 34 and 91 kDa proteins demonstrated a reverse profile and may not be a good vaccine candidate.
\end{abstract}

Conclusions: The preliminary data obtained in this study proposes the potential of some of the antigens in Leishmania diagnosis and for test of cure. Additionally, some antigens demonstrated good immunoprophylactic cytokine production through T cell-mediated immune response, suggesting future vaccine candidates for VL. However, further studies are necessary to explore these antigens in diagnosis and to access the long-term immune response.

\footnotetext{
*Correspondence: nali@iicb.res.in

†Sarfaraz Ahmad Ejazi and Smriti Ghosh contributed equally to this work

${ }^{1}$ Infectious Diseases and Immunology Division, Indian Institute

of Chemical Biology, Kolkata, West Bengal, India

Full list of author information is available at the end of the article
}

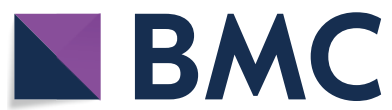

c The Author(s) 2020. This article is licensed under a Creative Commons Attribution 4.0 International License, which permits use, sharing, adaptation, distribution and reproduction in any medium or format, as long as you give appropriate credit to the original author(s) and the source, provide a link to the Creative Commons licence, and indicate if changes were made. The images or other third party material in this article are included in the article's Creative Commons licence, unless indicated otherwise in a credit line to the material. If material is not included in the article's Creative Commons licence and your intended use is not permitted by statutory regulation or exceeds the permitted use, you will need to obtain permission directly from the copyright holder. To view a copy of this licence, visit http://creativecommons.org/licenses/by/4.0/. The Creative Commons Public Domain Dedication waiver (http://creativecommons.org/publicdomain/zero/1.0/) applies to the data made available in this article, unless otherwise stated in a credit line to the data. 
Keywords: Biochemistry, Parasitology, Leishmaniasis, Immunology, Diagnosis, Vaccination, Th1/Th2, Cytokines

\section{Background}

Despite a reduction in the number of visceral leishmaniasis $(\mathrm{VL})$ cases in previously endemic areas, many regions still show the presence of disease burden and also spread into newer areas has been reported. Early diagnosis with complete treatment of cases, together with the development of a vaccine, should be considered as important solutions [1-3]. Clinical diagnosis of VL depends on the demonstration of parasites in spleen or bone marrow aspirates, a complicated procedure to perform, but still considered as the gold standard in routine diagnosis [4]. High levels of anti-leishmanial antibodies are developed during the acute disease, which is maintained up to several years and can be detected by various serological tests such as ELISA, IFAT and direct agglutination test (DAT). Development of the rK39 antigen-based strip test has brought a major advancement in VL diagnosis for practice in field settings. Nevertheless, the poor sensitivity of the rK39 in East Africa, and suboptimal performance in Brazil, necessitate the scope of research for better diagnostic tools [5]. Failure to differentiate between active and past infection is another limitation with the rK39 test. Since a single antigen is not effective for all endemic areas, newer antigens have been screened and evaluated for the serodiagnosis of VL. Recombinant leishmanial antigens such as rKLO8, rKE16 and rK28 are few of them [6-9]. In our previous studies, we have reported the diagnostic value of Leishmania promastigote membrane antigens (LAg) through various immunological techniques such as ELISA, immunoblot and dipstick test [5, $10,11]$. Moreover, anti-leishmanial antibodies in the sera of active and cured VL patients have shown variable reactivity against several proteins of LAg in the immunoblot assay [12].

Leshmania donovani infections in humans do not always result in disease manifestations. In VL-endemic areas self-resolving infection has also been observed by developing parasite-specific antibodies and/or $\mathrm{T}$ cell response [13, 14]. Furthermore, patients who have recovered from kala-azar are usually immune to reinfection, which suggests that vaccination against leishmaniasis should be feasible $[15,16]$. Studies from animal models have shown that protection against Leishmania can be achieved using parasite-specific proteins, DNA or genetically attenuated parasites $[17,18]$. Advances in our understanding of Leishmania-host interactions, Leishmania pathogenesis, protective immunity and the availability of the complete $L$. donovani genome sequence, could take this a step further. Reports from earlier studies in our laboratory have demonstrated the immunogenicity of $L$. donovani promastigote membrane antigens, either free or in liposomal preparations $[19,20]$. In addition to inducing very good protection in the murine model, it could induce remarkable lymphoproliferation and protective cytokines (IFN- $\gamma$ and IL-12) production in successfully treated kala-azar patients [21]. Similar results were also observed with soluble leishmanial antigens (SLA), partially purified from leishmanial membrane antigens, which when entrapped in cationic liposomes conferred almost complete protection as a prophylactic or therapeutic vaccine against $L$. donovani in BALB/C mice [22]. These data indicate that some of these peptides are more immunogenic than the others in experimental mouse models, and could be interesting to investigate their immunogenicity in humans.

Screening of the most immunodominant antigens of formerly tested purified antigens in response to the human immune system is an important task. On the other hand, differentiation between active and past infection is one of the major challenges for the serodiagnosis of VL. Moreover, antigens used in recent years are mostly recombinants that evade post-translational modification unlike purified antigens [23]. Therefore, in this study, we have evaluated purified leishmanial antigens, LAg and SLA, and electroeluted different fractions of LAg such as $31,34,36,45,51,63,72,91$ and $97 \mathrm{kDa}$ proteins in their native state to differentiate active VL from healthy controls and cured individuals, through ELISA. We have also characterized 31, 34, 51, 63, 72 and $91 \mathrm{kDa}$ proteins of LAg for immune stimulatory efficacy of PBMCs of cured VL patients through cytokine analysis as potential vaccine candidates.

\section{Methods}

\section{Sample collection}

Serum samples used in this study were collected from the School of Tropical Medicine (STM), Kolkata. Twentythree VL patients were enrolled for the longitudinal study having single dose liposomal amphotericin B therapy (10 $\mathrm{mg} / \mathrm{kg}$ ). Blood samples were collected before the treatment, from active VL cases (AVL), one month after the treatment, from cured VL cases $(\mathrm{CVL})$, and from followups (FU) at approximately six months post-treatment. Sera was also collected from 23 other symptomatically similar diseases comprised of four samples each from malaria, tuberculosis, pneumonia, typhoid and viral fever, and one sample from a patient with a liver abscess, a patient with systemic lupus erythematosus, and a 
patient with pancreatitis. Sample collection continued with 23 healthy individuals as controls from the Indian Institute of Chemical Biology (IICB), Kolkata.

\section{Parasite culture and purification of leishmanial antigens} Leishmania donovani strain AG83 (ATCC ${ }^{\odot}$ PRA-413 ${ }^{\mathrm{TM}}$ ) of the parasite was regularly maintained in hamsters. Amastigotes were isolated from sacrificed hamsters and allowed to transform into promastigotes in culture medium (M199) at $22{ }^{\circ} \mathrm{C}$ with supplements such as $10 \%$ heat-inactivated fetal bovine serum, $100 \mathrm{U} / \mathrm{ml}$ penicillin $\mathrm{G}$ sodium and $100 \mathrm{~g} / \mathrm{ml}$ streptomycin sulfate. Promastigotes were subcultured through fresh medium passages and 3rd to 5th passage cultures were harvested and centrifuged to obtain cell pellets. Cell pellets were then washed in PBS and stored at $-20{ }^{\circ} \mathrm{C}$ until use.

Leishmania promastigote antigens ( $\mathrm{LAg})$ were purified from the cell pellet. In a typical experiment, cells were suspended in $5 \mathrm{mM}$ Tris- $\mathrm{HCl}$ ( $\mathrm{pH}$ 7.4) and vortexed for $12 \mathrm{~min}$ ( $2 \mathrm{~min}, 6$ times) for the parasite membrane to become leaky. Parasites were then centrifuged $(2310 \times g$, $10 \mathrm{~min}, 4^{\circ} \mathrm{C}$ ) to collect the ghost membrane pellet which was then resuspended in the same buffer and subjected to ultrasonication ( $30 \mathrm{~s}, 6$ times). The suspension was centrifuged $\left(5190 \times g, 30 \mathrm{~min}, 4{ }^{\circ} \mathrm{C}\right)$ to obtain the antigens in the supernatant. The concentration of LAg was estimated by Lowry's methods and stored at $-80{ }^{\circ} \mathrm{C}$ until further use. Soluble leishmanial antigen, SLA, was also purified from Leishmania promastigote culture similar to LAg with some modifications. The cell pellet was suspended in $1 \mathrm{mM}$ EDTA, $5 \mu \mathrm{g}$ leupeptin, $1 \mathrm{mM}$ iodoacetamide and $1 \mathrm{mM}$ phenylmethylsulfonyl fluoride in $5 \mathrm{mM}$ Tris- $\mathrm{HCl}$ buffer, pH 7.4. The suspension was vortexed and centrifugation and sonication was performed as previously for LAg, followed by solubilisation in $(1 \% \mathrm{w} / \mathrm{v})$ octyl- $\beta$-Dglucopyranoside at $4{ }^{\circ} \mathrm{C}$ overnight. The next day the solubilised suspension was centrifuged at $100,000 \times \mathrm{g}$ for $1 \mathrm{~h}$. The supernatant collected contains SLA which was dialyzed and finally stored at $-80{ }^{\circ} \mathrm{C}$ after the protein concentration was estimated using Lowry's method.

\section{SDS-PAGE and electroelution}

Proteins were first denatured by the reducing agent $\beta$-mercaptoethanol ( $\beta \mathrm{ME}$ ), and resolved by $10 \%$ SDSPAGE. Different molecular weight proteins were separated from LAg (10 $\mu \mathrm{g} /$ lane) and SLA $(5 \mu \mathrm{g} /$ lane). The pattern of LAg and SLA proteins were visualized by Coomassie Blue. Rf values for the molecular weights of the respective proteins were determined by the automated Image Lab software (Bio-Rad, CA, USA) in comparison to the standards. For electroelution, 31, 34, 36, 45, 51, 63, 72, 91 and $97 \mathrm{kDa}$ protein bands were excised from the LAg gel and subjected to electroelution as per the manufacturer's protocol (Model-422; Bio-Rad). Subsequently, each protein was dialyzed against PBS. Electroeluted proteins after quantification were resolved on SDS-PAGE separately for reconfirmation of their molecular weights.

\section{Indirect ELISA for antibody detection}

Indirect ELISA to capture antibodies was performed on 96-well flat bottom plates (Maxisorp Nunc; Thermo Fisher Scientific, MA, USA). In brief, wells were coated with $1 \mu \mathrm{g} /$ well concentration of purified and electroeluted proteins with phosphate buffer $(100 \mu \mathrm{l} /$ well $)$ and incubated overnight in $4{ }^{\circ} \mathrm{C}$. The next day, antigen-coated wells were blocked with $1 \%$ BSA in PBS $(200 \mu \mathrm{l} /$ well $)$ for $2 \mathrm{~h}$ at $37{ }^{\circ} \mathrm{C}$. Subsequently, serum samples (1:2000) followed by peroxide conjugated antihuman IgG (1:4000) were applied to the wells in PBS buffer $(100 \mu \mathrm{l} /$ well $)$ and incubated for $1 \mathrm{~h}$ at $37{ }^{\circ} \mathrm{C}$. Plates were washed in each step with PBS and Tween 20 to remove any non-specific binding. Finally, wells were incubated in the substrate, o-phenylenediamine dihydrochloride (OPD), and $\mathrm{H}_{2} \mathrm{O}_{2}$ in the phosphate-citrate buffer $(50 \mu \mathrm{l} /$ well $)$. The biological reaction was stopped with sulfuric acid and optical density values were acquired by using an ELISA plate reader (RS232C; Thermo Fisher Scientific, MA, USA) at a wavelength of $492 \mathrm{~nm}$.

\section{Stimulation of PBMCs and cytokines analysis}

Peripheral blood mononuclear cells (PBMCs) from heparinized blood samples of one month cured VL patients and healthy individuals were isolated by density gradient centrifugation using Histopaque-1077 (SigmaAldrich, MO, USA) and finally resuspended in RPMI 1640 medium with serum supplements and antibiotics. PBMCs obtained from each individual were cultured in triplicate $\left(1 \times 10^{6}\right.$ cells/well $)$ with and without antigen stimulation. Stimulation of cured VL and healthy PBMCs with LAg $(12.5 \mu \mathrm{g} / \mathrm{ml})$ and cured VL PBMCs with electroeluted antigens $(1.5 \mu \mathrm{g} / \mathrm{ml}) 31,34,51,63$, 72 and $91 \mathrm{kDa}$, were performed at $37{ }^{\circ} \mathrm{C}$ in a $\mathrm{CO}_{2}$ incubator. Supernatant from the cultures were collected after $96 \mathrm{~h}$ and stored at $-20{ }^{\circ} \mathrm{C}$ for cytokine analysis. The level of cytokines, IFN- $\gamma$, IL-12, IL-10 and TGF- $\beta$, along with IFN- $\gamma /$ IL-10 and IFN- $\gamma /$ TGF- $\beta$, were measured by ELISA according to the manufacturers' instructions (BD OptEIA ELISA kit; BD Biosciences, NJ, USA). Briefly, capture antibodies specific to the cytokines were coated in the wells of 96-well plates in carbonate buffer overnight at $4{ }^{\circ} \mathrm{C}$. Subsequently, wells were blocked and incubated with culture supernatants for 1 h. Cytokine specific detection antibodies were used in the wells followed by TMB substrate. Optical density values were obtained in the ELISA reader at $450 \mathrm{~nm}$. 


\section{Mass spectrometry}

Protein bands of molecular masses, 34 and $45 \mathrm{kDa}$ were stained with Coomassie Blue and excised from 10\% SDSPAGE of LAg. The proteins imbibed in the gel bands were digested by an in-gel tryptic digestion kit, according to the protocol provided by the manufacturer (Thermo Fisher Scientific). Subsequently, co-crystallization of the digested peptides with the matrix was performed and subjected to MALDI-TOF/TOF for MS/MS spectra (Applied Biosystems, CA, USA). Data obtained from mass spectrometry were identified through the MASCOT search engine (http://www.matrixscience.com/ search_form_select.html).

\section{Statistics}

Statistical studies were conducted with Graph Pad Prism software version V. A two-tailed Student's t-test was performed for paired and unpaired samples from indirect ELISA. $P$-values less than 0.05 were considered significant with $95 \%$ confidence intervals. Cut-off values were determined by the receiver-operator curve (ROC), where $100 \%$ sensitivity was obtained. The difference in cytokine production was determined by the two-tailed unpaired Student's t-test.

\section{Results}

Immunoreactivity of LAg and SLA with Leishmania infected human serum samples

Leishmania promastigote membrane antigens (LAg) and soluble leishmanial antigens (SLA) were isolated from $L$. donovani promastigote culture and subjected to $10 \%$ polyacrylamide gel electrophoresis. LAg and SLA were resolved in their components of different molecular weights by SDS PAGE. LAg contains approximately 25-30 immunogenic proteins whereas SLA is a mixture of about ten dominant protein fractions (Fig. 1).

One of the most challenging objectives for Leishmania-specific antibody-based serodiagnosis is to differentiate an active VL patient from a patient with a past infection. In this regard reactivity of LAg and SLA with paired serum samples of $13 \mathrm{VL}$ patients before treatment, one month cured and patients followed-up after six months, were evaluated by ELISA. We observed that there was a comparatively higher antibody titer in the active VL sera against LAg in comparison to other study groups. However, cured VL patients, just after treatment, showed a significant decrease in antibody titer but $84.16 \%$ positivity in ELISA (Fig. 2a). In the follow-up group, sera after six months of treatment showed a remarkable decrease in the antibody titer with no positive reactivity against LAg and the mean was below the cut-off line. No cross-reaction of LAg was found with 13 other diseases and 13 healthy

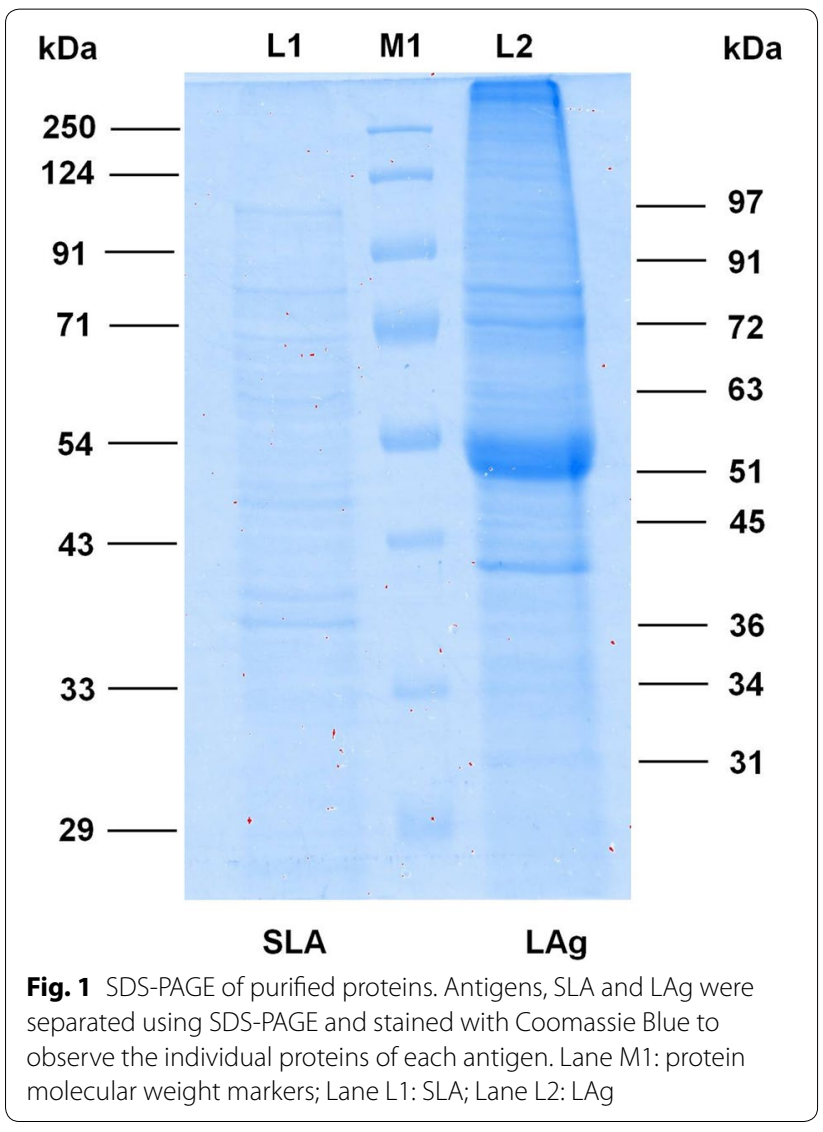

controls tested. A similar assay was also performed with SLA, where both active and one month cured VL patients showed $100 \%$ positivity with SLA (Fig. 2b). However, $7.69 \%$ of the follow-up patients were still positive with SLA. Healthy controls and other diseases, however, did not show cross-reactivity with SLA. These results reveal that LAg and SLA have the strong serodiagnostic potential for VL. Both LAg and SLA could not satisfactorily differentiate active VL from past infections following one month post-therapy. However, after six months of infection, antibody titers significantly decreased below the cut-off and became $100 \%$ negative for LAg. Therefore, in the next step, we isolated different components of LAg for immunoreactive studies of individual antigens.

\section{Electroelution of LAg antigens}

To access the immunoreactive potential of different protein fractions of LAg, nine polypeptides of LAg, 31, $34,36,45,51,63,72,91$ and $97 \mathrm{kDa}$ were eluted out electrophoretically from Coomassie Blue-stained gels and subjected to SDS-PAGE (Fig. 3). To differentiate active disease from cured VL and follow-up patients, ELISA was carried out with all nine electroeluted antigens. 

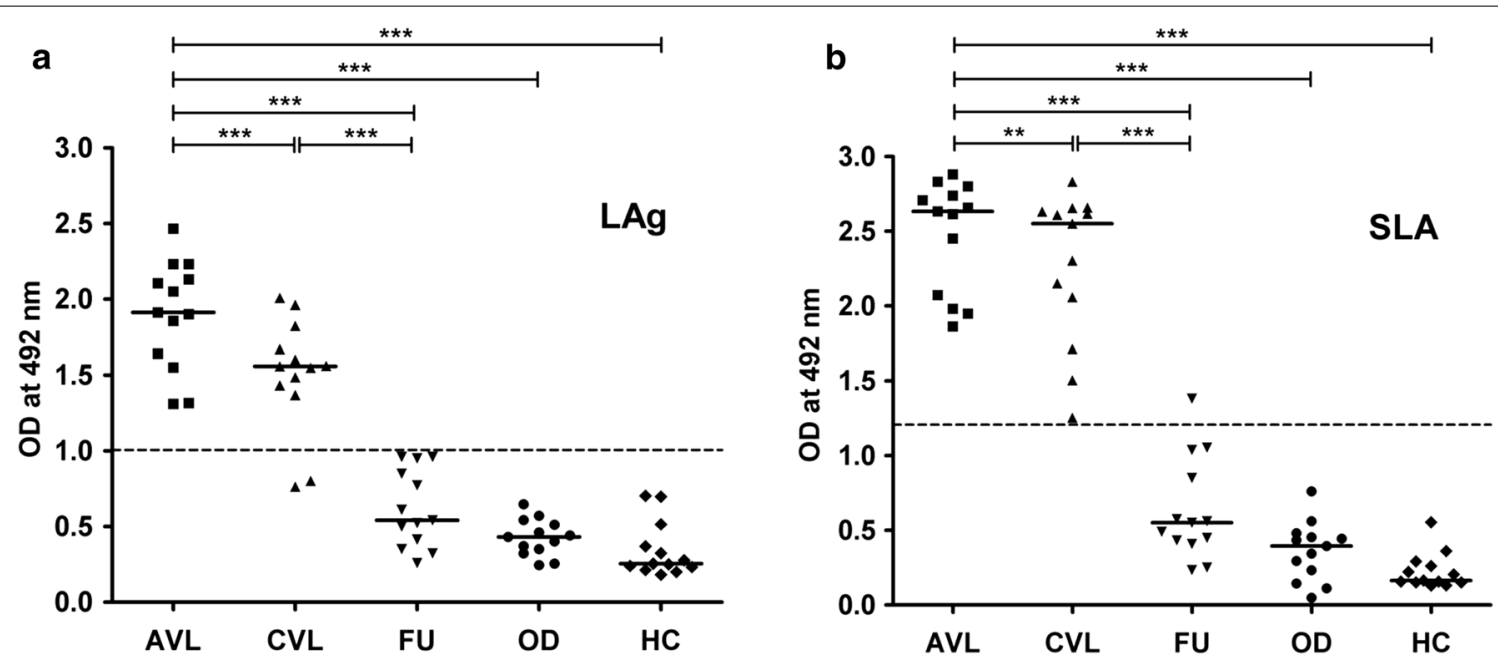

Fig. 2 Indirect ELISA using purified antigens LAg and SLA. Optical density values were obtained by ELISA for the detection of serum antibodies against antigens $\operatorname{LAg}(\mathbf{a})$ and SLA (b). Paired serum samples $(n=13)$ from confirmed active VL cases before the treatment (AVL), cured VL one month after treatment (CVL), and six-month follow-ups (FU) were investigated against purified leishmanial antigens, LAg and SLA. Serum samples from 13 healthy individuals (HC) and 13 from other diseases were also tested. The cut-off values were selected from the ROC curve where 100\% sensitivity and specificity were achieved. Each point represents an average of triplicate values obtained from a single sample

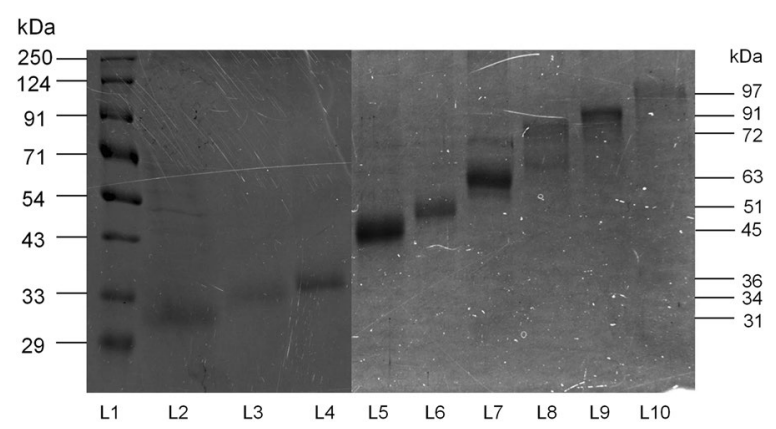

Fig. 3 SDS-PAGE of electroeluted proteins of LAg. Electroeluted antigens 31, 34, 36, 45, 51, 63, 72, 91 and 97 kDa were run on 10\% SDS-PAGE. Lane L1: marker; Lane L2: 31 kDa; Lane L3: 34 kDa; Lane L4: 36 kDa; Lane L5: 45 kDa; Lane L6: 51 kDa; Lane L7: 63 kDa; Lane L8: 72 kDa; Lane L9: 91 kDa; Lane L10: 97 kDa. The figure depicted here was obtained from two separate gels

\section{Reactivity of electroeluted proteins in ELISA}

Nine electroeluted antigens were evaluated in ELISA for their ability to distinguish the active disease from other diseases and healthy controls as well as from the cured $\mathrm{VL}$ and follow-up samples. Cut-off values were set for each eluted antigen where antibody titer above the cutoff line was considered as positive and below as negative. The cut-off values for VL positivity were obtained from the ROC curve, where maximum sensitivity and specificity were achieved in comparison with healthy controls. Out of the nine antigens, 100\% sensitivity with active VL sera was observed with all electroeluted antigens. The specificity of the antigens was calculated based on the cross-reactivity of the antigens with the healthy controls and other diseases. With the healthy control samples tested, the mean optical density values for all the electroeluted antigens were below the cut-off line. 31, 34, 36, 51 and $97 \mathrm{kDa}$ proteins were found to be $100 \%$ specific, whereas antigens 63 and $91 \mathrm{kDa}$ showed more than $90 \%$ specificity. The reactivity of eluted antigens with other diseases showed $100 \%$ specificity with 34 and $51 \mathrm{kDa}$ antigens, followed by 91 and $97 \mathrm{kDa}$ with $95.75 \%$ specificity. Therefore, for the purpose of diagnosis, 34 and 51 $\mathrm{kDa}$ proteins were found to be the best to distinguish active VL from healthy controls and other diseases. The percent sensitivity and specificity of all the electroeluted antigens are listed in Table 1.

The study to differentiate active VL from cured and past infections using electroeluted antigens demonstrated considerable differences among the patient groups (Fig. 4). There was a statistically considerable decline in serum IgG levels against all the antigens in the one month cured patient sera compared to their respective active disease sera. However, except for 63, 72 and $91 \mathrm{kDa}$, none of the antigens showed mean optical density values below its cut-off. Recognition of electroeluted antigens with sera after one month cure ranged from 8.69-100\% which is still positive and not ideal for the differentiation of the diseased state from cure. Followup patients who recovered from VL six months posttreatment showed a significant decline in the IgG levels against 31, 34, 51, 63, 72, and $91 \mathrm{kDa}$ antigens and their levels were comparable to the healthy control sera where 
Table 1 Sensitivity and specificity of purified and electroeluted antigens with human sera

\begin{tabular}{llllll}
\hline $\begin{array}{l}\text { Purified and } \\
\text { electroeluted } \\
\text { antigens }(\mathrm{kDa})\end{array}$ & $\begin{array}{l}\text { Sensitivity to active VL } \\
\text { patients in \% }(n / \mathrm{N})\end{array}$ & $\begin{array}{l}\text { Sensitivity to 1-month } \\
\text { treated VL patients in \% } \\
(n / \mathrm{N})\end{array}$ & $\begin{array}{l}\text { Sensitivity to 6-month } \\
\text { follow-up patients in \% } \\
(n / \mathrm{N})\end{array}$ & $\begin{array}{l}\text { Specificity to other } \\
\text { diseases in \% }\left(n^{\prime} / \mathrm{N}\right)\end{array}$ & $\begin{array}{l}\text { Specificity to } \\
\text { healthy samples in } \\
\%\left(n^{\prime} / \mathrm{N}\right)\end{array}$ \\
\hline $\mathrm{LAg}$ & $100(13 / 13)$ & $84.16(11 / 13)$ & $0(0 / 13)$ & $100(13 / 13)$ & $100(13 / 13)$ \\
SLA & $100(13 / 13)$ & $100(13 / 13)$ & $7.69(1 / 13)$ & $100(13 / 13)$ & $100(13 / 13)$ \\
31 & $100(23 / 23)$ & $60(14 / 23)$ & $26.08(6 / 23)$ & $39.1(9 / 23)$ & $100(23 / 23)$ \\
34 & $100(23 / 23)$ & $73.91(17 / 23)$ & $13.04(3 / 23)$ & $100(23 / 23)$ & $100(23 / 23)$ \\
36 & $100(23 / 23)$ & $69.56(16 / 23)$ & $78.26(18 / 23)$ & $40(8 / 23)$ & $100(23 / 23)$ \\
45 & $100(23 / 23)$ & $100(23 / 23)$ & $60.86(14 / 23)$ & $30.43(7 / 23)$ & $60.86(14 / 23)$ \\
51 & $100(23 / 23)$ & $91.30(21 / 23)$ & $30.43(7 / 23)$ & $100(23 / 23)$ & $100(23 / 23)$ \\
63 & $100(23 / 23)$ & $47.82(11 / 23)$ & $4.34(1 / 23)$ & $91.30(21 / 23)$ & $95.65(22 / 23)$ \\
72 & $100(23 / 23)$ & $39.1(9 / 23)$ & $0(0 / 23)$ & $65.21(15 / 23)$ & $73.91(17 / 23)$ \\
91 & $100(23 / 23)$ & $8.69(2 / 23)$ & $0(0 / 23)$ & $95.75(22 / 23)$ & $91.30(21 / 23)$ \\
\hline
\end{tabular}

Abbreviations: $\mathrm{n}$, no. of positive samples in each group; $\mathrm{N}$, total no. of samples tested in each group; $\mathrm{n}$, no. of negative samples in each group

the mean was below the cut-off. Amongst all the antigens, 72 and $91 \mathrm{kDa}$ showed the most promising results, with $100 \%$ negative reactivity in follow-up patients.

\section{Evaluation of electroeluted proteins for cytokine analysis from human PBMCs}

Immunity to leishmaniasis is known to depend on protective cellular responses against the parasites. In order to assess the immunogenicity of LAg and eluted proteins, $31,34,51,63,72$ and $91 \mathrm{kDa}$ antigens were used to stimulate the PBMCs of cured VL patients for the production of protective cytokines IFN- $\gamma$ and IL-12, and anti-inflammatory cytokines, IL-10 and TGF- $\beta$, which promote disease progression. IFN $-\gamma$ is the most dominant Th1 cytokine required to control Leishmania infection. Analysis of cytokines revealed variable levels of IFN- $\gamma$ production when PBMCs were stimulated with electroeluted antigens, whereas unstimulated cultures produced negligible IFN- $\gamma$ (Fig. 5a). Prominent levels of IFN- $\gamma$ were produced by the LAg-stimulated PBMCs of VL treated and healthy individuals as compared to unstimulated PBMCs. Significantly high levels of IFN- $\gamma$ were produced by 51 and $63 \mathrm{kDa}$ antigens followed by 31 and $72 \mathrm{kDa}$ antigens when compared with unstimulated cultures. In comparison, 34 and $91 \mathrm{kDa}$ antigens produced low levels of IFN- $\gamma$. IL-12 is another protective cytokine produced by the macrophages and in turn, activates the $\mathrm{T}$ cells to produce IFN- $\gamma$ and accelerates the leishmanicidal function. Healthy and treated PBMCs exhibited significant IL-12 induction against LAg (Fig. 5b). Similar to the LAg stimulated cultures, significantly elevated levels of IL-12 were produced by 51 and $63 \mathrm{kDa}$ antigens followed by 31 and $72 \mathrm{kDa} .34$ and $91 \mathrm{kDa}$ antigens produced comparatively lower levels of IL-12.
IL-10 and TGF- $\beta$ are two immunosuppressive cytokines that promote parasite survival and help in disease progression in VL infection. Our study demonstrated that LAg stimulated PBMCs from VL treated and healthy subjects produced moderate levels of IL-10. Among electroeluted antigens, 34 and $91 \mathrm{kDa}$ antigens showed maximum levels of IL-10. In contrast, comparatively low levels of IL-10 were produced by 31 , 51, 63 and $72 \mathrm{kDa}$ electroeluted antigens (Fig. 5c). Low levels of TGF- $\beta$ were produced by the healthy PBMCs than the cured PBMCs against LAg, whereas 31, 51, 63 and $72 \mathrm{kDa}$ antigens produced median levels of TGF- $\beta$ by the treated PBMCs (Fig. 5d). Again, 34 and $91 \mathrm{kDa}$ antigens produced the highest levels of TGF- $\beta$ similar to that produced by LAg stimulated cultures (Fig. $5 \mathrm{~d}$ ). Ratios of IFN- $\gamma$ and IL-10 as well as IFN- $\gamma$ and TGF- $\beta$ were plotted in Fig. 5e, $\mathrm{f}$.

\section{Identification of electroeluted proteins}

Out of nine proteins of LAg evaluated in this study, except 34 and $45 \mathrm{kDa}$ proteins, all proteins were identified in the earlier reports (Table 2). Therefore, we identified the remaining two proteins through MALDI-TOF mass spectrometry and matched with the Leishmania sequence. The $34 \mathrm{kDa}$ protein was identified as Leishmania analogue of the receptors of activated $C$ kinase (LACK) whereas the $45 \mathrm{kDa}$ protein was $\beta$ tubulin (Additional file 1: Figure S1, Additional file 1: Figure S2).

\section{Discussion}

Visceral leishmaniasis is one of the foremost fatal infectious diseases for which no effective vaccine is currently available. Moreover, there is scope for finding better and effective tests for its diagnosis and to access treatment 

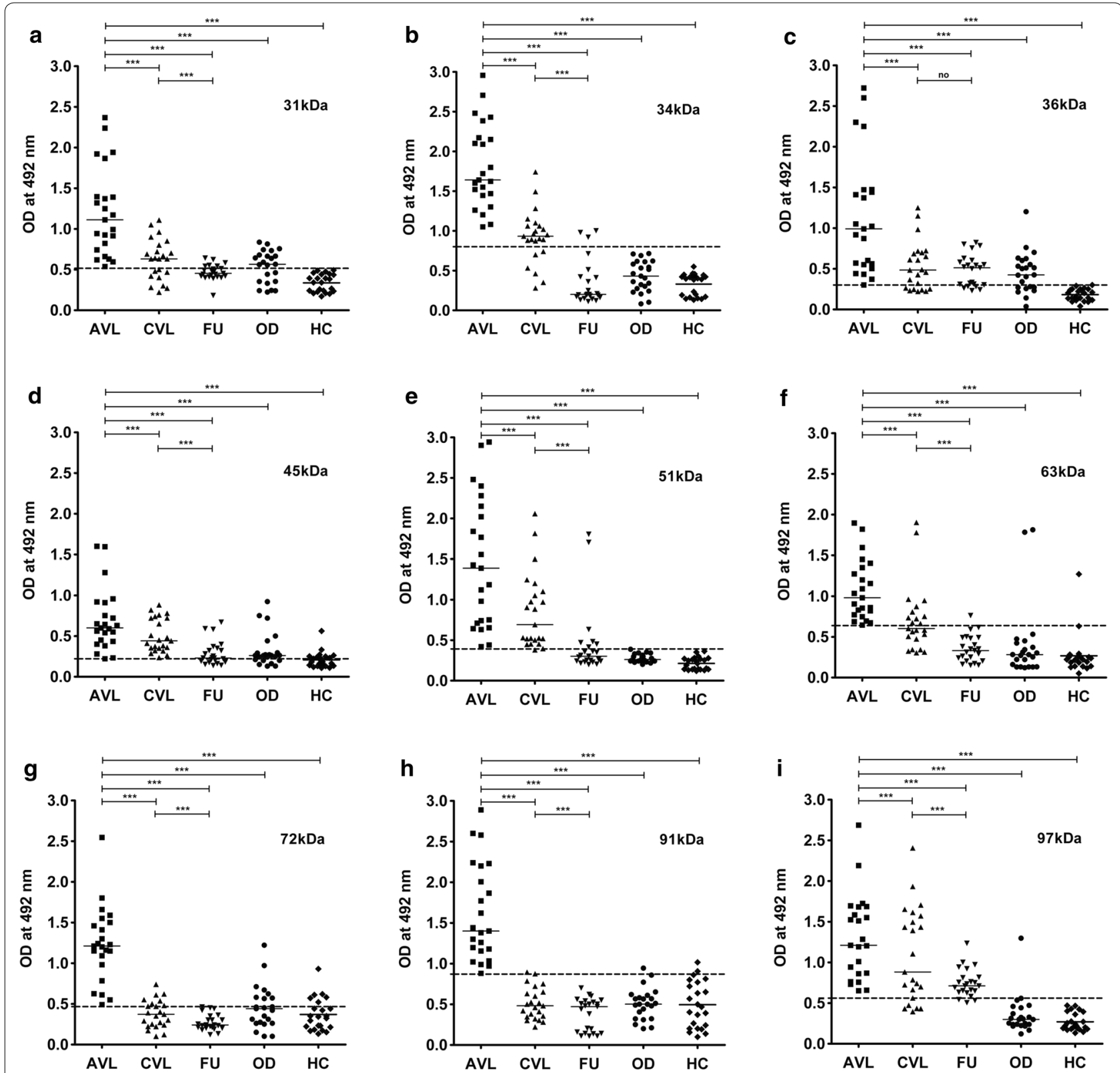

Fig. 4 Indirect ELISA using electroeluted antigens. IgG reactivity of eluted antigens 31 (a), 34 (b), 36 (c), 45 (d), 51 (e), 63 (f), 72 (g), 91 (h) and 97 $\mathrm{kDa}$ (i) with serum samples from $23 \mathrm{VL}$ patients at three different time points that is active VL before the treatment (AVL), after one month cured VL $(\mathrm{CVL})$, and after six months follow-up cases (FU). The study also included sera from symptomatically similar other diseases (OD; $n=23)$ and healthy controls $(\mathrm{HC} ; n=23)$. The horizontal lines denote the mean value for each group. The dotted lines denote the cut-off values selected from the ROC curve where maximum sensitivities and specificities were achieved. Each point represents an average of triplicate values obtained from a single sample

response. In this study, we have isolated and evaluated several Leishmania promastigote membrane antigens which can be used as probable candidates for diagnosis, test of cure as well as for vaccine development.

Estimating parasites microscopically from tissue aspirates is considered the gold standard for diagnosis but bears the risk of internal bleeding, pain, and dependence on a trained person is the major hurdle. VL is also characterized by the production of Leishmania-specific antibodies during disease. Hence in the last two decades, several serological methods have been evaluated largely using recombinant antigens with varying degrees of sensitivity and specificity. However, defined antigens do not undergo post-translational modifications such 


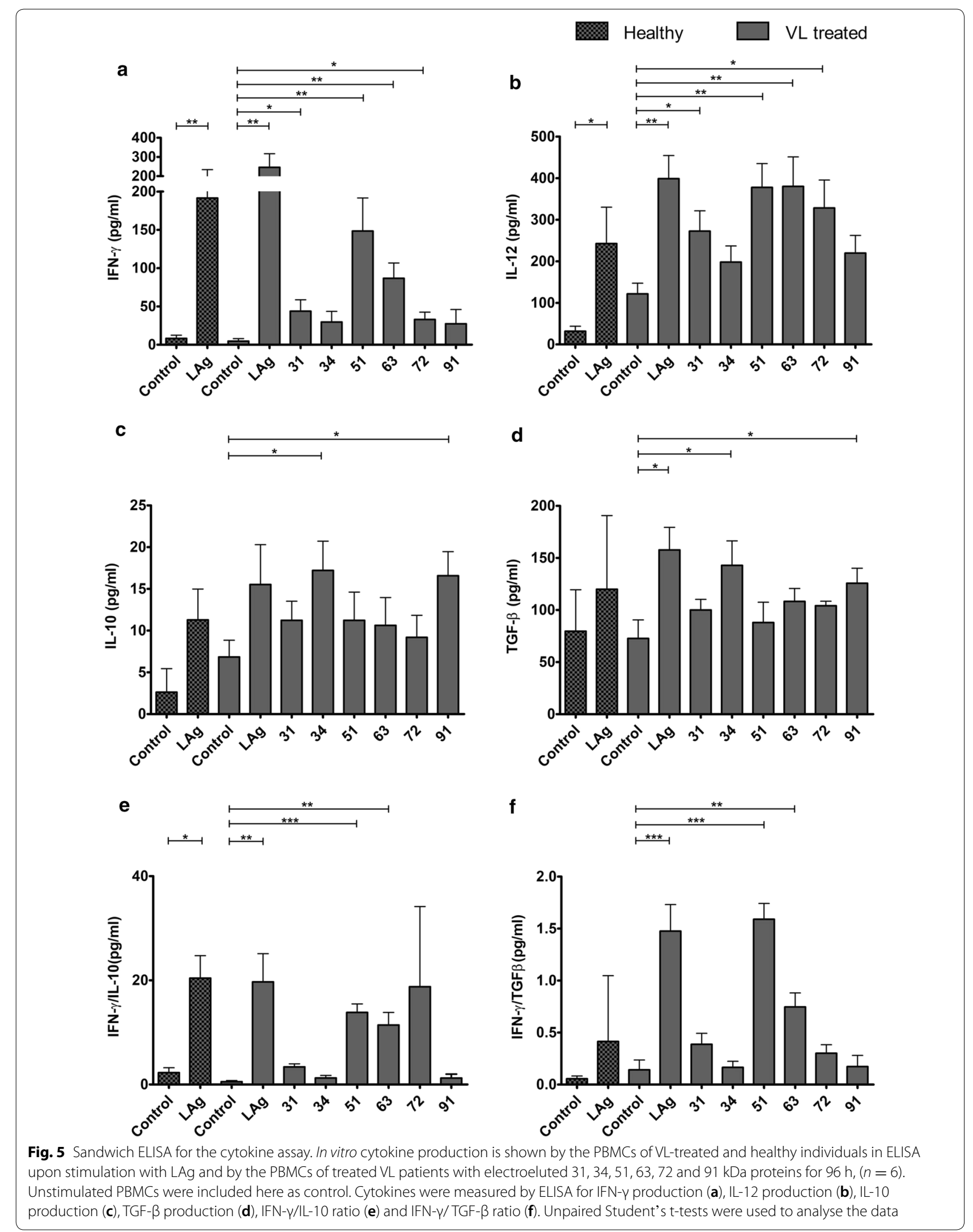


Table 2 List of electroeluted fractions of LAg and corresponding proteins

\begin{tabular}{|c|c|c|}
\hline Electroeluted proteins $(\mathrm{kDa})$ & Protein name & References \\
\hline 31 & ATP synthase a chain & [29] \\
\hline 34 & Leishmania analogue of the receptors of activated C kinase (LACK) & Present study \\
\hline 36 & Elongation factor $1-a$ & [31] \\
\hline 45 & $\beta$ tubulin & Present study \\
\hline 51 & $\beta$ tubulin & [29] \\
\hline 63 & Glycoprotein & [24] \\
\hline 72 & Heat shock 70-related protein 1 mitochondrial precursor (HSP 70) & [29] \\
\hline 91 & $\beta$ tubulin & [29] \\
\hline 97 & Not yet indentified from LAg/ may be nucleoporins-93 (NUP-93) & [35] \\
\hline
\end{tabular}

as glycosylation. Therefore, these antigens lack carbohydrate and lipid moieties as present in the native state of the antigen [23]. Thus, antigens in their native state were electroeluted to validate their diagnostic properties. Additionally, most of the serological tests are for the diagnosis of active VL conditions; therefore, assessing treatment efficacy remains an important challenge for $\mathrm{VL}$ management and elimination. In the present study, two purified leishmanial antigens, LAg and SLA, together with nine electroeluted proteins of LAg were evaluated for serodiagnosis of VL as well as to differentiate active VL from past infection. LAg has previously been reported to demonstrate a strong potential for the diagnosis of VL by detecting antigen-specific antibodies in serum and urine samples [10, 24]. SLA has also been reported in vaccine-mediated protection in murine VL $[22,25]$. However, there is no study to investigate the different components of these purified antigens separately for diagnosis and to investigate their reactivity during different treatment phases. Herein, we observed that there is a significant decline in the Leishmania-specific antibody titers in the patients' sera after one month of treatment for both LAg and SLA, However, they were still positive at the set cut-off values. Sera collected after six months of treatment demonstrated a sharp decline in the antibody titers against both LAg and SLA. Antibody levels for SLA remained above the cut-off line in some patients after six months follow-up in contrast to LAg, where at this time point, antibodies decreased to the level below the cut-off. Therefore, absolute differentiation between active VL sera from past infections is possible with LAg. In search of more defined immunoreactive antigens of LAg, nine antigens, 31, 34, 36, 45, 51, 63, 72, 91 and 97 $\mathrm{kDa}$, were electroeluted to study their reactivity individually. We found that few electroeluted antigens introduced improvement over total antigen LAg, some of which hold a strong potential for serodiagnosis of VL. Antigens 31, 34, 36, 51 and $97 \mathrm{kDa}$ were found to be $100 \%$ sensitive to detect active VL cases with $100 \%$ specificity against healthy controls. Antigens 34 and $51 \mathrm{kDa}$ differentiated active VL and other diseases with $100 \%$ specificity. On the basis of the overall sensitivity and specificity of the antigens, these two antigens could be prospective candidates for future serodiagnosis of VL.

In kala-azar therapy, the initial cure is determined just after completion of treatment and a definite cure is determined six months post-treatment. Therefore, it is more important to distinguish the active disease from past infections of VL after at least six months of therapy. Very few studies have been undertaken to distinguish active VL from past infections of VL using serum samples. Antibody levels in sera were found to fall in response to rK26 and rK18 antigens after six months of treatment [26]. Studies of Indian and Sudanese L. donovani patient sera with crude antigen showed a significant decrease in IgG1 antibody levels after six months of cure [27]. A recent study with rK39 antigen demonstrated a greater decline in IgG1 isotypes in follow-up patients than IgG [28]. Therefore, to distinguish the active disease from past infections of VL, an antigen with high sensitivity for active VL may or may not be a good candidate for use in a test of cure. In our study, we were to some extent successful in differentiating active disease from past infections of VL using the electroeluted antigens. Interestingly, serum samples after six months of cure did not show reactivity with 72 and $91 \mathrm{kDa}$ antigens. The other antigens which also hold a strong potential to distinguish active VL from past infections were 34 and $63 \mathrm{kDa}$ proteins, and all these antigens had high reactivity with diseased VL sera and low reactivity with follow-up patient sera.

Most of the proteins electroeluted in this study have been identified through MALDI MS/MS in earlier reports [24, 29]. The role of these parasitic proteins in disease progression has been ascertained in mouse models from several previous studies [29-35]. The $31 \mathrm{kDa}$ protein was identified as ATP synthase $\alpha$ chain of Leishmania [29]. ATP synthase complex subunits are functionally associated with the membrane and thus can be 
potential targets for drugs, diagnostic probes or vaccine components against Leishmania. A protein with a molecular weight of $34 \mathrm{kDa}$ was identified in this study through mass spectrometry and recognized as a Leishmania analogue of the receptors of activated C kinase (LACK). LACK has been reported to induce a strong parasite-specific immune response and protects experimental mice against $L$. donovani infection [30]. The $36 \mathrm{kDa}$ protein was identified as elongation factor $1-\alpha(E F 1-\alpha)$, a translation factor that induces a cellular proliferative response and imparts long term immunity [31]. The $45 \mathrm{kDa}$ protein was identified in the present study as beta-tubulin. The $51 \mathrm{kDa}$ protein was also identified as beta-tubulin in our earlier reports, which exhibits high levels of protective cytokines and reduced infection in mice [29]. The 63 $\mathrm{kDa}$ protein is a membrane-anchored glycoprotein that corresponds to the infective stage and virulence of the parasite [32]. Another $72 \mathrm{kDa}$ protein belongs to a set of highly evolutionary conserved heat-shock proteins. They behave as chaperones and induced Th1-type of cellular responses in cured patients and hamsters [33, 34]. The 91 $\mathrm{kDa}$ protein showed homology with $\beta$-tubulin but did not show immunogenicity and protective immunity as compared to the other tubulins in the experimental L. donovani vaccine study [29]. The $97 \mathrm{kDa}$ protein was recently identified in both promastigote and amastigote forms of parasite as nucleoporins 93 (NUP93) and found to be an immunoprophylactic agent against Leishmania [35].

Identification of key antigenic targets of the protective human immune response against VL is central to the development of an efficacious vaccine against Leishmania. Cell-mediated immune responses are the key determinants for the natural course of Leishmania infection. Although human VL is known to elicit a mixed Th1 and Th2 response, protective immunity is achieved when the cell-mediated Th1 response is predominant. Thus, antigens that predominantly stimulate the Th1 response are considered as potential protective antigens [36]. The IL-12 driven IFN- $\gamma$ dominated Th1 response is associated with resistance to infection against Leishmania, as it has a direct effect on the macrophage microbicidal response and other effector killing mechanisms [37]. Conversely, antigens that predominantly stimulate the Th2 response from these cells have been regarded as of lesser interest as vaccine candidates because they are likely to be associated with pathology [38]. IL-10 and TGF- $\beta$ are the major immunosuppressive cytokines in VL and they can modulate macrophages and $\mathrm{T}$ cell functions and promote multiplication of Leishmania parasites as evident in patients with VL [21].

Studies have evaluated purified and recombinant proteins as well as whole parasite lysate for potential immunostimulatory activity in experimental models. Hence, it is important to evaluate leishmanial antigens for cellular immune responses in humans. Our earlier report reveals that entrapment of LAg in the cationic liposomes conferred significant levels of protection against infection in hamsters and $\mathrm{BALB} / \mathrm{c}$ mice [39]. Immunodominant proteins of LAg, 31, 51, 63, 72 and $91 \mathrm{kDa}$ antigens elicited a protective immune response in an experimental model [29]. Since PBMCs derived from VL-treated patients were exposed to Leishmania infection they showed an increase in protective cytokine response against LAg in the present study. However, PBMCs derived from healthy individuals also showed similar results suggesting the role of LAg antigens in prophylaxis. We characterized the immune response through cytokine analysis of six electroeluted antigens of LAg, 31, 34, 51, 63, 72 and 91 $\mathrm{kDa}$ that were recognized by sera of active and cured $\mathrm{VL}$ patients. Although the six antigens were able to stimulate PBMCs from cured VL individuals, the response was quite variable. The 51 and $63 \mathrm{kDa}$ antigens induced maximum elevation of protective Th1 cytokines IFN- $\gamma$ and IL-12, similar to that of LAg, followed by 31 and 72 $\mathrm{kDa}$ antigens that produced comparatively lower levels of IFN- $\gamma$ and IL-12. Antigens 34 and $91 \mathrm{kDa}$, however, did not show significant production of IFN- $\gamma$ and IL-12. Lower levels of immunosuppressive cytokines IL-10 and TGF- $\beta$ were secreted by $31,51,63$ and $72 \mathrm{kDa}$ antigens. In contrast, 34 and $91 \mathrm{kDa}$ antigens produced significantly higher levels of the suppressive cytokines, comparable to LAg. Therefore, these two antigens could be the reason for high IL-10 and TGF- $\beta$ production from LAg. Moreover, the ratio of IFN- $\gamma / \mathrm{IL}-10$ and IFN- $\gamma /$ TGF- $\beta$ of treated patients also demonstrated the protective immune profile against $\mathrm{LAg}$ and 51 and $63 \mathrm{kDa}$ among electroeluted antigens.

From this study, 51 and $63 \mathrm{kDa}$ antigens may be considered to be the most potent vaccine candidates based on the cytokine analysis. The 31 and $72 \mathrm{kDa}$ antigens were found to be less immunogenic and 34 and $91 \mathrm{kDa}$ antigens did not show any potential to be considered as a vaccine candidate. Although the diagnostic and immunoprophylactic responses of antigens were clearly described in these preliminary experiments, the number of samples used was small. We therefore consider this as a limitation of our work and will try to overcome this in the next phase of the study.

\section{Conclusions}

We report here the evaluation of electroeluted Leishmania membrane proteins some of which showed high sensitivity for active VL cases, as well as ability to differentiate from past VL infection. Thus they can be used as probable diagnostic candidates as well as for test of cure. 
Moreover, some of the electroeluted antigens stimulate significant protective cytokines IFN- $\gamma$ and IL-12, and low levels of disease progressive cytokines, IL-10 and TGF$\beta$, from human PBMCs after cure. Therefore, they may be considered as potential subunit vaccine candidates against VL.

\section{Supplementary information}

Supplementary information accompanies this paper at https://doi. org/10.1186/s13071-020-04138-7.

Additional file 1: Figure S1. MALDI-TOF spectra of the tryptic fragments obtained from the peptide $34 \mathrm{kDa}$. Figure S2. MALDI-TOF spectra of the tryptic fragments obtained from the peptide $45 \mathrm{kDa}$.

\section{Abbreviations}

VL: visceral leishmaniasis; HIV: human immunodeficiency virus; LAg: Leishmania promastigote membrane antigens; SLA: soluble leishmanial antigen; ELISA: enzyme-linked immunosorbent assay; DAT: direct agglutination test; IFAT: immunofluorescence antibody test; MALDI-TOF: matrix assisted laser desorption/ionization-time of flight; SDS-PAGE: sodium dodecyl sulfate-polyacrylamide gel electrophoresis; EHC: endemic healthy control; NEHC: non-endemic healthy control; OD: other diseases; $\mathrm{HC}$ : healthy control; $\mathrm{AVL}$ : active visceral leishmaniasis; CVL: cured visceral leishmaniasis; FU: follow-up patients.

\section{Acknowledgments}

Not applicable.

\section{Authors' contributions}

SAE, SG and NA conceived and designed this study and wrote the manuscript. SAE and SG carried out the experiments. AB, MK and SD provided experimental support, analysed results and reviewed the manuscript. SB conducted the MALDI experiment for the $34 \mathrm{kDa}$ protein. MR and RPG contributed in clinical sample collection, conducted confirmatory tests and provided ethical clearance from the hospitals. All authors read and approved the final manuscript.

\section{Funding}

This study has, in part, received funding from UK Research and Innovation via the Global Challenges Research Fund under grant agreement 'A Global Network for Neglected Tropical Diseases' grant number MR/P027989/1, Sir J. C. Bose Fellowship, India and Fellowships from the Council of Scientific and Industrial Research, India.

\section{Availability of data and materials}

All data generated or analysed during this study are included in this published article and its additional file.

\section{Ethics approval and consent to participate}

Approval for sample collection and research was obtained from the Institutional Ethical Committee, CSIR-Indian Institute of Chemical Biology (IICB) and the School of Tropical Medicine (STM), Kolkata, India. The study was conducted according to the guidelines and suggestions set by the committees. Patients involved in this study were informed before sample collection and written consent was obtained.

\section{Consent for publication}

Not applicable.

\section{Competing interests}

The authors declare that they have no competing interests.

\section{Author details}

${ }^{1}$ Infectious Diseases and Immunology Division, Indian Institute of Chemical Biology, Kolkata, West Bengal, India. ${ }^{2}$ Present Address: Department of Botany, Serampore College, Hooghly, Serampore, West Bengal, India.

${ }^{3}$ Present Address: Dr. Kanailal Bhattacharyya College, Dharmatala, Ramrajatala,
Santragachi, Howrah, West Bengal, India. ${ }^{4}$ Department of Tropical Medicine, School of Tropical Medicine, Kolkata, West Bengal, India.

Received: 24 December 2019 Accepted: 14 May 2020

Published online: 30 May 2020

\section{References}

1. Burza S, Croft SL, Boelaert M. Leishmaniasis. Lancet. 2018;392:951-70.

2. Lindoso JAL, Moreira CHV, Cunha MA, Queiroz IT. Visceral leishmaniasis and HIV coinfection: current perspectives. HIV AIDS (Auckl). 2018:10:193-201.

3. Singh OP, Gedda MR, Mudavath SL, Srivastava ON, Sundar S. Envisioning the innovations in nanomedicine to combat visceral leishmaniasis: for future theranostic application. Nanomedicine (Lond). 2019;14:1911-27.

4. Ejazi SA, Ali N. Developments in diagnosis and treatment of visceral leishmaniasis during the last decade and future prospects. Expert Rev Anti Infect Ther. 2013:11:79-98.

5. Ejazi SA, Bhattacharya P, Bakhteyar MA, Mumtaz AA, Pandey K, Das VN, et al. Noninvasive diagnosis of visceral leishmaniasis: development and evaluation of two urine-based immunoassays for detection of Leishmania donovani infection in India. PLoS Negl Trop Dis. 2016;10:e0005035.

6. Sivakumar R, Dey A, Sharma P, Singh S. Expression and characterization of a recombinant kinesin antigen from an old Indian strain (DD8) of Leishmania donovani and comparing it with a commercially available antigen from a newly isolated (KE16) strain of L. donovani. Infect Genet Evol. 2008;8:313-22.

7. Abass E, Bollig N, Reinhard K, Camara B, Mansour D, Visekruna A, et al. rKLO8, a novel Leishmania donovani-derived recombinant immunodominant protein for sensitive detection of visceral leishmaniasis in Sudan. PLoS Negl Trop Dis. 2013;7:e2322.

8. Pattabhi S, Whittle J, Mohamath R, El-Safi S, Moulton GG, Guderian JA, et al. Design, development and evaluation of rK28-based point-of-care tests for improving rapid diagnosis of visceral leishmaniasis. PLoS Negl Trop Dis. 2010;4:e822.

9. Bhargava P, Singh R. Developments in diagnosis and antileishmanial drugs. Interdiscip Perspect Infect Dis. 2012;2012:626838.

10. Saha S, Goswami R, Pramanik N, Guha SK, Saha B, Rahman M, et al. Easy test for visceral leishmaniasis and post-kala-azar dermal leishmaniasis. Emerg Infect Dis. 2011;17:1304-6.

11. Ejazi SA, Ghosh S, Saha S, Choudhury ST, Bhattacharyya A, Chatterjee M, et al. A multicentric evaluation of dipstick test for serodiagnosis of viscera leishmaniasis in India, Nepal, Sri Lanka, Brazil, Ethiopia and Spain. Sci Rep. 2019;9:9932.

12. Ravindran R, Anam K, Bairagi BC, Saha B, Pramanik N, Guha SK, et al. Characterization of immunoglobulin $\mathrm{G}$ and its subclass response to Indian kala-azar infection before and after chemotherapy. Infect Immun. 2004;72:863-70.

13. Duthie MS, Raman VS, Piazza FM, Reed SG. The development and clinical evaluation of second-generation leishmaniasis vaccines. Vaccine. 2012;30:134-41.

14. van Griensven J, Diro E. Visceral leishmaniasis: recent advances in diagnostics and treatment regimens. Infect Dis Clin North Am. 2019:33:79-99.

15. De Brito RCF, Cardoso JMO, Reis LES, Vieira JF, Mathias FAS, Roatt BM, et al. Peptide vaccines for leishmaniasis. Front Immunol. 2018;9:1043.

16. Olliaro PL, Shamsuzzaman TA, Marasini B, Dhariwal AC, Be-Nazir A, Mondal $D$, et al. Investments in research and surveillance are needed to go beyond elimination and stop transmission of Leishmania in the Indian subcontinent. PLoS Negl Trop Dis. 2017;11:e0005190.

17. Moafi M, Rezvan H, Sherkat R, Taleban R. Leishmania vaccines entered in clinical trials: a review of literature. Int J Prev Med. 2019;10:95.

18. Bi K, Chen Y, Zhao S, Kuang Y, John Wu CH. Current visceral leishmaniasis research: a research review to inspire future study. Biomed Res Int. 2018;2018:9872095.

19. Mazumdar T, Anam K, Ali N. A mixed Th1/Th2 response elicited by a liposomal formulation of Leishmania vaccine instructs Th1 responses and resistance to Leishmania donovani in susceptible BALB/c mice. Vaccine. 2004:22:1162-71. 
20. Saha S, Mazumdar T, Anam K, Ravindran R, Bairagi B, Saha B, et al. Leishmania promastigote membrane antigen-based enzyme-linked immunosorbent assay and immunoblotting for differential diagnosis of Indian post-kala-azar dermal leishmaniasis. J Clin Microbiol. 2005;43:1269-77.

21. Saha S, Mondal S, Ravindran R, Bhowmick S, Modak D, Mallick S, et al. IL-10- and TGF-beta-mediated susceptibility in kala-azar and postkala-azar dermal leishmaniasis: the significance of amphotericin B in the control of Leishmania donovani infection in India. J Immunol. 2007; 179:5592-603.

22. Bhowmick S, Ravindran R, Ali N. Leishmanial antigens in liposomes promote protective immunity and provide immunotherapy against visceral leishmaniasis via polarized Th1 response. Vaccine. 2007;25:6544-56.

23. Kuhne V, Rezaei Z, Pitzinger P, Buscher P. Systematic review on antigens for serodiagnosis of visceral leishmaniasis, with a focus on East Africa. PLoS Negl Trop Dis. 2019;13:e0007658.

24. Ejazi SA, Bhattacharyya A, Choudhury ST, Ghosh S, Sabur A, Pandey K, et al. Immunoproteomic identification and characterization of Leishmania membrane proteins as non-invasive diagnostic candidates for clinical visceral leishmaniasis. Sci Rep. 2018;8:12110.

25. Bhowmick S, Ravindran R, Ali N. gp63 in stable cationic liposomes confers sustained vaccine immunity to susceptible BALB/C mice infected with Leishmania donovani. Infect Immun. 2008;76:1003-15.

26. Vallur AC, Tutterrow YL, Mohamath R, Pattabhi S, Hailu A, Abdoun AO, et al. Development and comparative evaluation of two antigen detection tests for visceral leishmaniasis. BMC Infect Dis. 2015;15:384.

27. Bhattacharyya T, Ayandeh A, Falconar AK, Sundar S, El-Safi S, Gripenberg MA, et al. IgG1 as a potential biomarker of post-chemotherapeutic relapse in visceral leishmaniasis, and adaptation to a rapid diagnostic test. PLoS Negl Trop Dis. 2014;8:e3273.

28. Mollett G, Bremer Hinckel BC, Bhattacharyya T, Marlais T, Singh OP, Mertens $P$, et al. Detection of immunoglobulin $\mathrm{G} 1$ against rK39 improves monitoring of treatment outcomes in visceral leishmaniasis. Clin Infect Dis. 2019;69:1130-5.

29. Bhowmick S, Ali N. Identification of novel Leishmania donovani antigens that help define correlates of vaccine-mediated protection in visceral leishmaniasis. PLoS ONE. 2009:4:e5820.

30. De Oliveira Gomes DC, Schwedersky RP, Barbosa De Melo LD, Da Silva Costa Souza BL, De Matos Guedes HL, Lopes UG, et al. Peripheral expression of LACK-mRNA induced by intranasal vaccination with
PCI-NEO-LACK defines the protection duration against murine visceral leishmaniasis. Parasitology. 2012;139:1562-9.

31. Sabur A, Bhowmick S, Chhajer R, Ejazi SA, Didwania N, Asad M, et al. Liposomal elongation factor-1alpha triggers effector CD4 and CD8 T cells for induction of long-lasting protective immunity against visceral leishmaniasis. Front Immunol. 2018;9:18.

32. Kaur T, Sobti RC, Kaur S. Cocktail of gp63 and Hsp70 induces protection against Leishmania donovani in BALB/C mice. Parasite Immunol. 2011;33:95-103.

33. Rasouli M, Karimi MH, Kalani M, Ebrahimnezhad S, Namayandeh M, Moravej A. Immunostimulatory effects of Leishmania infantum HSP70 recombinant protein on dendritic cells in vitro and in vivo. Immunotherapy. 2014;6:577-85.

34. Jaiswal AK, Khare P, Joshi S, Kushawaha PK, Sundar S, Dube A. Th1 stimulatory proteins of Leishmania donovani: comparative cellular and protective responses of rtriose phosphate isomerase, rprotein disulfide isomerase and relongation factor- 2 in combination with $\mathrm{rHSP} 70$ against visceral leishmaniasis. PLoS ONE. 2014;9:e108556.

35. Singh MK, Jamal F, Dubey AK, Shivam P, Kumari S, Pushpanjali, et al. Visceral leishmaniasis: a novel nuclear envelope protein 'ucleoporins-93 (NUP-93)' from Leishmania donovani prompts macrophage signaling for T-cell activation towards host protective immune response. Cytokine. 2019;113:200-15.

36. Gillespie PM, Beaumier CM, Strych U, Hayward T, Hotez PJ, Bottazzi ME. Status of vaccine research and development of vaccines for leishmaniasis. Vaccine. 2016;34:2992-5.

37. Rodrigues V, Cordeiro-da-Silva A, Laforge M, Silvestre R, Estaquier J. Regulation of immunity during visceral Leishmania infection. Parasit Vectors. 2016;9:118.

38. Jain K, Jain NK. Vaccines for visceral leishmaniasis: a review. J Immunol Methods. 2015:422:1-12.

39. Bhowmick S, Mazumdar T, Sinha R, Ali N. Comparison of liposome based antigen delivery systems for protection against Leishmania donovani. J Control Release. 2010;141:199-207.

\section{Publisher's Note}

Springer Nature remains neutral with regard to jurisdictional claims in published maps and institutional affiliations.
Ready to submit your research? Choose BMC and benefit from:

- fast, convenient online submission

- thorough peer review by experienced researchers in your field

- rapid publication on acceptance

- support for research data, including large and complex data types

- gold Open Access which fosters wider collaboration and increased citations

- maximum visibility for your research: over $100 \mathrm{M}$ website views per year

At BMC, research is always in progress.

Learn more biomedcentral.com/submissions 